\title{
LITTÉRATURE
}

IRENA FILIPOWSKA

Université Adam Mickiewicz

\section{LA VISION TRAGIQUE DU MONDE DE ROGER MARTIN DU GARD PRÉSENTÉE DANS SES ROMANS}

\begin{abstract}
Filipowska Irena, La vision tragique du monde de Roger Martin du Gard présentée dans ses romans [Tragic vision of the world of Roger Martin du Gard based on his novels], Studia Romanica Posnaniensia, Adam Mickiewicz University Press, Poznań, vol. XXVII: 2001, pp. 3-10. ISBN 83232-1039-X, ISSN 0137-2475.

The tragic vision of the world, in the opinion of RMG consisted in ascertaining that, in the universe, neither God nor any metaphysical conception took any place. In this understanding the world is absurd and the presence of man in this world is pointless as well. A human being thrown on his/her own resources, surrounded by enemy forces - the biological fatality, that is to say diseases, old age and death, and the historical fatality in the form of wars weigh on, is always abandoned.
\end{abstract}

Roger Martin du Gard est un des plus éminents écrivains français vivant à cheval sur le $\mathrm{XIX}^{\text {eme }}$ et le $\mathrm{XX}^{\text {eme }}$ siècle. Son oeuvre se compose de romans (Devenir, Jean Barois, Les Thibault), de nouvelles (Confidence africaine et Vieille France), de pièces de théâtre (Le Testament du Père Leleu, La Gonfle et Le Taciturne) éditées dès les premières années du $\mathrm{XX}^{\text {ème }}$ siècle et à l'époque de l'entre-deux guerres. Tandis que son roman inachevé: Le Lieutenant-colonel de Maumort, ses nombreuses correspondances et son Journal ont été édités après sa mort, à partir de l' année 1968 et entre les années 1982-1993.

Né en 1881 et mort en 1958, Roger Martin du Gard (RMG) a été par conséquent, témoin de deux guerres mondiales et il a assisté à de profonds changements de vie en France et dans le monde. En 1937 le prix Nobel de littérature lui a été attribué surtout pour ses opinions pacifistes insérées dans Les Thibault, au moment où l'on sentait l'approche d'un conflit mondial.

Toutefois, d'après un bref aperçu sur les monographies et les articles qui lui ont été consacrés au cours de la $\mathrm{I}^{\text {ere }}$ moitié du $\mathrm{XX}^{\mathrm{e}}$ s., on s'était rendu compte que RMG et son oeuvre durant sa vie ont été peu connus. C'est seulement après l'attribution du 
prix Nobel de littérature que les Français se sont aperçus que leur connaissance de l'écrivain et de son oeuvre a été minime'.

Tandis qu'après la parution des oeuvres posthumes de RMG l'intérêt que l'on porte à ses écrits s'accroît d'années en années. Des colloques internationaux ont lieu dans différents pays qui réunissent les spécialistes de la production littéraire de l'écrivain et qui analysent aussi son oeuvre posthume. Ainsi sous le haut patronat du Ministre d'Etat et du Ministre des Affaires Etrangères et du Centre International de la Recherche sur RMG eurent lieu en 1990, à Bellême, 3 Journées Culturelles RMG ayant comme titre: «Guerre et Paix dans l'oeuvre de RMG».

En outre à la demande générale de.nombreux chercheurs français et étrangères, réunis à l'occasion de ce colloque, on a pu créer les «Cahiers RMG» afin de maintenir entre eux des relations durables ainsi que de diffuser auprès d'un large public les recherches actuelles sur RMG. Il est très intéressant de citer ici quelques titres d'articles consacrés aux oeuvres posthumes ou aux problèmes traités par RMG dans son Journal: a) Etre, apparaître, agir - RMG et le Lieutenant-colonel de Maumort $^{2}$; b) Maumort et Thésée, testaments de RMG et Gide $^{3}$; c) RMG et la formation intellectuelle des adolescents d'après la Corr. Générale ${ }^{4}$; d) La Correspondance $R M G$ - André Gide vue par un Soviétique en $1990^{5}$; e) $R M G$ témoin et juge du nazisme $e^{6}$;) Hardi Martin, continue R. Martin du Gard, le pacifisme et les pacifistes ${ }^{7}$.

On peut y ajouter encore un article consacré à RMG et la II ${ }^{\mathrm{e} m e}$ guerre mondiale d'après son Journal paru dans Scripta manent de $1998^{8}$ et écrit par nous.

La lecture alternative de la Correspondance de RMG échangée avec les hommes de lettres, les artistes et les amis les plus proches nous permet de nous rendre compte de son importance. Cette Correspondance est un document très précieux pour la connaissance de l'époque, pour la connaissance de la littérature et la connaissance de la mentalité de l'écrivain. Mais, d'après notre avis, c'est surtout son Journal, tenu du $\mathrm{I}^{\text {er }}$ juillet 1919 au 31 décembre 1949 auquel l'éditeur de

1 Pour une meilleure connaissance du problème cf. l'article de: I. Filipowska Etat de recherches se rapportant à l'oeuvre de RMG, Studia Romanica Posnaniensia, 1980, vol. 7.

${ }^{2}$ Fainas-Wehrmanne, Etre, paraitre, agir. RMG et le «Lieutenant-colonel de Maumort». Actes du Colloque International de Nice, 1992, Gallimard, Paris 1990, p. 128-139.

${ }^{3}$ A. Alessandri, Maumort et Thésée - testaments de RMG et Gide. Actes du Colloque International de Nice, Gallimard, Paris, p. 156-171.

4 J.C. Airal, RMG et la formation intellectuelle des adolescents d'après la Correspondance Générale. Cahier RMG 2, Gallimard, Paris 1991, p. 88-104.

${ }^{5}$ F. Narkiner, La Correspondance RMG - A. Gide vue par un Soviétique en 1990 RMG. Actes du Colloque International de Nice, 1990, Gallimard, Paris 1992, p. 127-139.

${ }^{6}$ M. Parra, I. Alba, RMG, témoin et juge du nazisme. L'Ecrivain et son Journal. Cahiers de RMG 5, Gallimard, Paris 1996.

${ }^{7}$ W. Offenstadt, Hardi Martin continue. RMG, le pacifisme et les pacifistes. Cahiers RMG, Gallimard, Paris 1994, p. 121-136.

${ }^{8}$ I. Filipowska, RMG et la $I I^{e ̀ m e}$ guerre mondiale d'après son Journal. Scripta Manent. Wydział Neofilologii UAM, Poznań 1998, p. 265-279. 
l'oeuvre en question a ajouté des documents intimes de RMG qui ont dépassé la date de 1949, qui est sans nul doute, le document des plus importants afin de connaître les problèmes de tous genres avec lesquels devaient lutter les hommes de l'époque contemporaine à RMG et RMG lui-même.

RMG considère le contenu du Journal: «comme simples jalons plantés sans art. En bordure de sa vie, mais qui doit être un véritable trésor de pensées et d'idées de tous genres». Le climat politique et spirituel d'une époque est un facteur très important dans la formation de la mentalité de chaque individu ainsi que dans la création des oeuvres d'un écrivain.

RMG qui est l'auteur de deux grands romans, cités déjà par nous à savoir de Jean Barois, édité en 1913 et des Thibault, qui sont un roman fleuve, se composant de 7 parties et d'un Epilogue dont la redaction a commencé en 1920 et qui s'est terminée en 1940, a présenté les héros dans des situations pleines de conflits. Cependant ce qui a particulièrement préoccupé RMG dans les 2 romans, cités ci-dessus, ce fut le tragique de la destinée humaine, problème se rangeant parmi les premiers dans son oeuvre. Néanmoins, ce problème n'a pas été traité d'une manière très approfondie dans les monographies consacrées à notre écrivain. C'est seulement notre thèse de doctorat portant le titre de: Le tragique de l'individu et de la collectivité dans les romans de RMG qui a analysé ledit problème à fond, soutenu en 1966 et qui a été édité plus tard en $1968^{\circ}$.

L'analyse détaillée de ce problème confrontée à la lecture du Journal a prouvé clairement l'existence du thème en question non seulement dans les oeuvres littéraires, mais surtout présent dans la pensée de notre écrivain. Cette tragique attitude envers le monde et les hommes a été conditionnée par plusieurs facteurs à savoir: par le climat de l'époque dans laquelle vivait l'écrivain pleine de conflits et de crises de toutes sortes ce qui a été relevé déjà par nous plus haut, de sa mentalité et de ses principes artistiques. Une corrélation existait entre eux. La vision tragique du monde est, par conséquent, une structure, laquelle, permettra de mieux comprendre l'essence des manifestations des sentiments humains et de leurs faits. Mais la vision du monde n'est pas faite seulement de données empiriques, mais, elle est, dans une certaine mesure aussi un outil de travail, une forme qui se compose de plusieurs éléments techniques dramatiques qui permettent de mettre en relief l'engagement des individus dans la vie politique et sociale. Sans aucun doute il ne faut pas oublier non plus, la personnalité de l'écrivain, et son attitude envers la réalite qui l'entoure, ce qui a été déjà avancé aussi par nous. On ne peut pas non plus ne pas prendre en considération son attitude envers Dieu et s'il ne croît pas en Lui, ce qui Le remplace, ses liens avec le monde qui l'entoure et ses liens avec les hommes. RMG ressentait très fortement la situation tragique dans laquelle vivaient ses contemporains. Il tâchait de resoudre tous les problèmes de la vie, mais il les resolvait toujours sous l'angle de l'individu. De là provient la disproportion dans la

9 I. Filipowska, Le tragique de l'individu, Poznań, Wydawnictwo Naukowe UAM, 1968, p. 105 et I. Filipowska, Le tragique de la collectivité dans les romans de RMG, Studia Romanica Posnaniensia, Poznań 1971, p. 65-77. 
présentation des individus et des collectivités sociales surtout dans son roman-fleuve Les Thibault. Les conflits métaphysiques, la lutte avec le «moi» de l'homme présentent autant de visions tragiques qui, comme nous le savons, ont été traité par Lucien Goldmann et qui sont des problèmes tragiques du monde et de l'homme étroitement liés entre eux et se rapportant à la croyance, ou à la négation de Dieu.

S'il s'agit des personnages, RMG les représente dans l'évolution historique des événements bien liés au passé et aux temps présents, certains annoncent même les temps futurs.

Face au mystère de l'Univers et face à l'énigme de la vie humaine l'homme doit faire le choix de son attitude envers la vie. Quand aux problèmes religieux et moraux le choix devra se faire après de longues réflexions et souvent il pourra être la cause de conflits tragiques. Face aux conflits et aux cataclysmes sociaux l'homme est obligé de choisir entre une attitude contemplative ou entre une attitude de réflexion ou entre une attitude de participation active. Par conséquent son attitude sera ou active ou passive. Dans la famille des Thibault les deux frères Jacques et Antoine devront choisir, face à la guerre, leur façon d'agir. Jacques va représenter une attitude active, difficile mais qui lui permettra d'agir d'après sa conscience et, le deuxième - Antoine, se fera entraîner par le déroulement des événements et prendra une attitude passive, conformiste, toutefois il se rendra compte qu'il n'agit pas bien, qu'il ne remplit pas bien son devoir.

En analysant les faits et les comportements des personnages de RMG nous comprenons que leur tragique dépendra de leurs attitudes envers les phénomènes de la vie et de la condition humaine. La personnalité de l'individu en contact avec une situation extérieure va créer, dans la majeure partie des cas, des situations lesquelles vont contenir des éléments tragiques. L'intensité du tragique dépendra du caractère des individus s'adaptant plus ou moins bien aux situations de la vie, à l'époque de crises. On peut classer alors les personnages tragiques de RMG: en conformistes, en résignés et en non-conformistes c'est-à-dire en révoltés.

Le tragiques des conformistes sera causé soit par leur caractère, soit par leur appartenance à un groupe social, lequel ne leur permettra pas de développer leur personnalité. Les conformistes se soumettent aux lois morales déterminées depuis longtemps, aux systèmes politiques, aux règlements d'une classe sociale, ou bien ils se soumettent à la volonte de leurs supérieurs, aux personnalités plus fortes qu'eux, guidés par le besoin de se plier aux règlements traçant leur conduite vu par l'impossibilité de vivre en dehors d'eux. On peut ranger parmi eux Mme Barois, le prêtre Scherts et le prêtre Levys. Les deux derniers n'ont pas été d'accord avec l'attitude des autorités de l'Eglise, toutefois, ils n'ont pas été capables de rompre avec l'institution à laquelle ils ont été traditionnellement liés. Ils ressentaient le besoin de continuer de croire en Dieu et ne savaient pas vivre en dehors d'une vie déterminée par les croyances religieuses, mais en même temps, ils comprenaient que la science qui se developpait niait l'existance de beaucoup de dogmes.

La connaissance interne de ces contradictions, la mauvaise conscience provenant de la conviction d'une personnelle faiblesse, le manque de courage afin 
de tirer des conclusions conformes à l'analyse de la situation, tous ces éléments stigmatisent une empreinte d'un conflit tragique aux personnages de RMG présentés plus haut. D'autres ont été des conformistes par indifférence, par fainéantise intellectuelle, tel était Antoine Thibault, lequel, ne s'intéressait qu'à son métier de médecin et à sa vie privée. D'autres encore étaient des conformistes par scepticisme et par fatalité tel fut le cas du dr Philip; d'autres encore par orgueil et par esprit de conformité comme ce fut le cas d'Oscar Thibault et pour terminer cette liste des conformistes il nous faut citer $\mathrm{M}$. Chasle et Mlle de Weize qui se soumettaient au destin par simple faiblesse. Le tragique des conformistes a été causé par la constatation de la nécessité de se libérer du conformisme vu l'évolution des conditions de vie et de la dévaluation de toutes leurs conceptions vitales, mais la majeure partie d'entre eux, n'avaient pas assez de forces de le faire, ils se rendaient pleinement compte du manque de volonté de leur part. D'autres encore sous l'influence d'événements extérieurs violents comprenaient que la route qu'ils avait choisie pendant toute leur vie, a été non seulement mauvaise mais également funeste pour eux-même et pour autrui aussi. Leur attitude passive était capable de créer des malheurs collectifs.

Il y avait des moments où ils se rendaient clairement compte qu'ils sont totalement isolés de la vie et séparés de ceux auxquels ils s'étaient soumis et c'est à ce moment-là qu'ils comprenaient pleinement le tragique de la situation dans laquelle ils se trouvaient.

Dans les romans de RMG les individus résignés souffraient beaucoup eux aussi. La résignation est une variante du conformisme. Certains individus se soumettaient au destin sans lutte, étant donné que la vie avait déçu leurs espérances, ou bien quand ils pensaient que la vie était meilleure et moins cruelle. Ce genre de tragique est un tragique de la capitulation. Nicole Héquet s'était justement trouvée dans une telle situation. Le tragique du renoncement peut être causé par le manque d'une forte volonté afin de pouvoir mener une vie libre et pleinement personnelle.

Ces individus sont incapables d'aucun effort et ils sont condamnés à l'échec. Gise de Weize en est le meilleur exemple. La certitude de son impuissance sera la source de ses tragiques souffrances.

La constatation de l'inutilité du renoncement au bonheur personnel et la certitude que les nombreux sacrifices n'ont donné aucun résultat positif et quelquefois même, au contraire, ont eu un résultat négatif, représente un autre genre de tragique des résignés. Pour le pasteur Grégory qui s'est consacré avec la plus grande joie à faire du bien aux autres, la pensée de l'inefficacité de son propre renoncement représentait la note tragique qui rongeait son âme secrètement.

Le tragique aspect de la résignation peut provenir aussi d'une lutte ou d'une révolte de longue durée terminée par une défaite. Les mobiles de l'attitude résignée de Mme de Fontanin vont être divers et complexes. C'est surtout le fatalisme qui ne lui permettait pas de lutter efficacement afin de réaliser ses propres idéaux, elle se rendait également compte qu'elle avait mal placé ses sentiments surtout s'il s'agissait de son mari. Sa vie se composait de constantes inquiétudes. Tout en se rendant 
parfaitement compte de la situation dans laquelle elle se trouvait, néanmoins, elle n'avait ni assez de force, ni assez de volonté pour y faire face et pour lutter efficacement avec l'état de choses qui était le sien. Ces contradictions intérieures qu'elle ne savait pas combattre l'ont rendue malheureuse pour toute sa vie. Le tragique des résignés était le résultat de la constatation de leur propre impuissance ou bien il était causé par la constatation de l'inefficacité de l'absolu renoncement fait au nom du bien suprême. Dans leur cas plus le renoncement était extrême, plus la souffrance tragique était intense.

Chez RMG le groupe le plus nombreux se composait d'individus révoltés pour lesquels la vie était une suite de conflits et de souffrances. Les individus révoltés tâchaient de se libérer des collectivités sociales qui les limitaient, qui les diminuaient. Ces individus tâchaient de connaître le mieux leur propre personnalité afin de pouvoir choisir le groupe social qui leur conviendrait le mieux. Leur tragique dependra aussi bien de leur mentalité que du groupe social avec lequel ils ont rompu, ou bien, du groupe auquel ils se sont inscrits et qui ne leur convenait pas non plus. Après la libération beaucoup d'entre eux n'auront pas assez de force pour rester fidèles aux idéaux de liberté pour lesquels ils ont combattu et pour lesquels ils se sont à nouveau laisser subjuguer. Cet état de choses leur fera comprendre que leur tragique situation est une situation qui est le résultat de leur faiblesse et qui fait d'eux des esclaves. Tel fut surtout le tragique de Jean Barois.

RMG présente des personnages qui, en cherchant leur authentique «moi» seront continuellement en conflit avec eux-mêmes. La collectivité n'exercera pas sur eux une influence prépondérante. Tel a été le tragique de Jenny de Fontanin. En la personne de Jacques Thibault RMG présente le héros par excellence tragique. Son tragique exprime la difficulté de vivre d'un individu avide de justice, de sincérité et surtout de liberté, en continuelle révolte contre la société et les collectivités qui étouffaient le plus souvent ces valeurs. L'analyse de sa vie a demontré les multiples aspects de son tragique, à savoir: le tragique individuel conditionné par sa mentalité, le tragique de la condition humaine, le tragique historique auquel on pourrait appliquer l'adjectif «pathétique», étant donné que Jacques Thibault a vécu à l'époque de profondes transformations politiques et sociales. La source de son tragique a été la perte de la foi en l'idéal auquel il avait dévoué sa vie, la constatation de l'échec de sa révolte, l'inutilité du sacrifice de son propre bonheur et de son propre avenir. Sa mort tragique est par conséquent la fin logique de toute son existence.

Le tragique de l'individu dans le monde de RMG était basé, entre autres, sur les conflits qui existaient entre les individus et les collectivités. Cependant il ne porte son attention que sur les individus, et la collectivité ne l'intéresse que dans la mesure où elle joue un rôle dans la vie de ces individus. Dans ses romans RMG a analysé à fond deux collectivités: la famille et le groupement de jeunes révolutionnaires genevois. Les autres collectivités sont à peine mentionnées; il les admet comme un fait acquis sans les reconstruire et les analyser de plus près (l'Eglise, le groupement militaire, politique, professionnel, de camaraderie, la classe 
sociale). Le tragique des individus vivant en communauté est causé par le manque de tolérance réciproque, les membres d'une famille souffrent très souvent également de la réduction de leur liberté. Aucun membre de la famille Barois, Thibault ou Fontanin ne possédait le sentiment de la communauté des buts et des idéaux vitaux.

La source d'une telle constatation tragique due à l'impossibilité de vivre en pleine tolérance familiale, présentée dans les romans de RMG, proviendrait, sans nul doute, de sa vie privée, car il luttait constamment au sein de sa propre famille, avec le difficile caractère de sa femme Hélène et de sa fille Christiane ce qui le rendait vraiment malheureux. Déjà en 1923, le 15 janvier, il écrivait dans son Journal: «Ma présence entre Hélène et Christiane est de plus en plus nécessaire. Ces deux caractères sont inconciliables et se torturent l'un l'autre. Tout cela est triste, triste. [...] J'assiste impuissant, à des incompatibilités essentielles» ${ }^{10}$.

Au cours des années la mésentente entre RMG et sa femme s'était encore aggravée. Le 22 juillet 1937 il a écrit, toujours dans son Journal, que: «L'air du Tertre est devenu irrespirable. [...] Ma joie de vivre est, une fois de plus, empoisonnée par Hélène. [...] C'est si bête ce gâchage de notre paix familial. [...] On est prisonnier d'une sorte d'envoûtement [...] Je suis sans courage devant cette nouvelle crise» ${ }^{11}$.

Quand aux groupes de jeunes révolutionnaires genevois, tous appartenaient à la

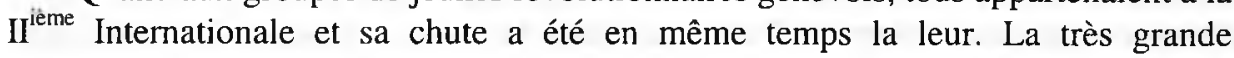
divergeance se rapportant à la ligne de conduite et à une méthode qui permettrait d'atteindre le but commun, c'est-à-dire l'exécution du programme de la II $^{\text {rème }}$ Internationale, la surestimation de la sociale démocratie qui a trahi ses propres principes et a sous-estimé les forces du nationalisme très fortement ancrées dans l'esprit des individus, ce furent autant de causes de sa désagrégation. La décomposition d'une collectivité projette le tragique sur les individus liés avec elle.

Dans l'univers de RMG ni la métaphysique ni Dieu n'occupent aucune place. Pour lui le monde n'a pas de sens et la présence de l'homme sur ce monde n'en a pas non plus aucun. RMG nous paraît être, dans un certain sens, un existentialiste. Pour lui l'homme sans aucune aide métaphysique voué à ses propres forces, assailli de toutes parts par les phénomènes incompris qui, très souvent, lui sont hostiles, sur lequel pèse la fatalité biologique sous forme de maladies, de vieillesse et de mort, le fatalisme historique sous forme de guerres, est toujours isolé. Seul, il lutte avec de tragiques conflits intérieurs et la société à laquelle il s'adresse ne le comprend pas.

Pendant toute sa vie RMG a été persuadé de l'absurdité de la condition humaine. Dans une de ses lettres inédite du 20 mars 1943 adressées à son ami Felix Sartiaux il lui a ecrit: «J'ai cherché en vain un sens à la vie, un but à la vie, à la condition humaine et je sens maintenant qu'on m'enterrera bredouille. Il est bien

${ }^{10} \mathrm{RMG}$, Journal II. Gallimard, Paris 1993, p. 361.

${ }^{11}$ RMG, Journal 111, Gallimard, Paris 1993, p. 69. 
probable que l'homme n'a pas de destin en ce monde ni en aucun autre. C'est dommage, un univers moins incohérant, moins absurde serait plus confortable. Mais je préfère encore ma déception aux fausses consolations de billevésées méthaphysiques. Je crois avoir très complètement exprimé mon point de vue à la page 322 de l'Epilogue. Je ne me rendais pas bien compte, en l'écrivant, à quel point, je résume là ma «conclusion». Cette conclusion résumant la philosophie vitale de RMG et sa tragique vision du monde est la suivante: «Mais plus on réfléchit, plus on a regardé autour de soi, en soi, et plus on est pénétré par cette vérité évidente: «Ça ne rime à rien». Des milliers d'êtres se forment sur la croûte terrestre, y grouillent un instant, puis se décomposent et disparaissent, laissant la place à d'autres millions qui, demain, se désagregeront à leur tour. Leur courte apparition ne «rime» à rien. La vie n'a pas de sens. Et rien n'a d'importance si ce n'est de s'efforcer à être le moins malheureux possible au cours de cette éphémère villégiature» ${ }^{12}$.

Toutefois, celui qui admetterait que dans ce monde il n'y a pas de place à l'espoir et à l'énergie se tromperait. La vie de l'homme, d'après RMG, n'a pas de sens, oui, mais cette tragique constatation ne doit pas lui enlever le courage de lutter avec les contrariétés, ni avec l'espoir d'une lente évolution de l'humanité. Chaque homme libéré d'illusions métaphysiques devrait transmettre à la postérité ce qu'il a obtenu lui-même, mais amélioré et enrichi par lui. Il pourra le faire uniquement dans un commun et fraternel effort en vaincant, au moins, une partie de son tragique sort. Dans une lettre, insérée dans son Journal, adressée à sa femme Hélène du 4 janvier 1940 il lui fait part d'un projet concernant l'argent qu'il devra toucher des droits d'auteur sur l'Epilogue qui représente à peu près une vingtaine ou une trentaine de mille francs. Il voudrait les donner à une oeuvre du Secours national. Voilà ce qu'il projette de faire: «Je ne voudrais pas que cet argent aille à une oeuvre de défense nationale, je veux dire d'armement. Et j'ai pensé au Secours national qui est une oeuvre de guerre pour laquelle les journaux font de la propagande. (...) Le but est d'alléger les détresses résultant de l'état de guerre pour les populations civiles de venir en aide aux femmes et aux enfants, aux vieillards que la guerre a mis dans la misère» ${ }^{13}$.

La pensée de vouloir secourir les personnes se trouvant dans une situation dramatique correspond à sa philosophie vitale basée sur la volonté de diminuer le sort tragique des hommes en le leur allégeant.

Cette attitude d'entraide humanitaire ressemble à l'attitude de Camus exprimée dans la Peste. Le dr Rieux s'efforce, lui aussi, d'aider les hommes se trouvant dans une situation tragique.

En terminant ce bref aperçu concernant la vision tragique du monde de RMG ne voyons-nous pas certaines analogies avec la vision qui on pourrait avoir de notre monde d'aujour d'hui au seuil du $3^{\text {eme }}$ millenaire?

\footnotetext{
${ }^{12}$ RMG, Les Thibault - Epilogue, Gallimard, Paris 1955, p. 988.

${ }^{13}$ RMG, Journal III, Gallimard, Paris 1993, p. 312.
} 